\title{
Flavour mixing and mass matrices via anticommuting properties
}

\author{
R Delbourgo $\dagger$ \\ † School of Mathematics and Physics, University of Tasmania, Private Bag 37 GPO \\ Hobart, AUSTRALIA 7001 \\ E-mail: Bob.Delbourgo@utas.edu.au
}

\begin{abstract}
Five anticommuting property coordinates can accommodate all the known fundamental particles in their three generations plus more. We describe the points of difference between this scheme and the standard model and show how flavour mixing arises through a set of expectation values carried by a single Higgs superfield.
\end{abstract}

Submitted to: J. Phys. A: Math. Gen.

PACS numbers: $11.10 \mathrm{Kk}, 11.30 . \mathrm{Hv}, 11.30 . \mathrm{Pb}, 12.10 .-\mathrm{g}$ 


\section{Property from anticommuting coordinates}

The standard model appears to be on a firm footing with many experimental verifications of particle states, interactions and decays. Yet there is some uneasiness in the physics community about the plethora of couplings and masses (that are tied to 20 or more parameters) and a lack of understanding about the 'generation problem'. Symmetry schemes which link the three families have been proposed aplenty and they attempt to explain the character of flavour mixing in weak interactions by invoking various relations between the mixing parameters due to some assumed underlying idea, such as permutation subsymmetries, texture zeros, seesaw effects, etc. Great efforts have gone into justifying each proposal but none stands out and certainly no consensus has been reached on the correct description, even though the $V_{U D}$ matrix elements are quite circumscribed today, in contrast to $V_{L N}$. Thus one is led to investigate alternative approaches which may be more predictive.

Recently we have proposed a scheme [1] where five fundamental anticommuting (complex) coordinates $\zeta^{\mu}$-appended to the four (real) space-time ones $x^{m}$ - are linked to something tangible, namely flavour or property. The framework therefore describes the where, when and what of an event. Fundamental particle properties are composites [2, 3] corresponding to polynomials in $\zeta$ and are of course limited because of property anticommutativity. Another nice feature is the effective reduction in 'dimension' due to the well-known cancellation between fermionic and bosonic degrees of freedom. We outlined a general relativistic extension in which gravity fell into the $x-x$ sector, gauge fields in the $x-\zeta$ sector and the Higgs field in the $\zeta-\zeta$ sector; however we shall not develop that side here. Rather we will focus on the particle aspects of the scheme, trying comprehend how the weak flavour mixing comes about and attempting to fix the mixing parameters with resulting quark and lepton masses.

It is worth stressing that our scheme differs in a number of respects from the standard model and these will be amplified in the next sections. The main difference is that there are more than 3 generations - a feature of other models too. However in our scheme the third and fourth coloured generations have quarks which are not weak isodoublet but isotriplet, with accompanying quarks $X$ having fermion number $F=1 / 3$ and charge $Q=-4 / 3$. This might be considered as an awful prediction, but considering how little one knows about the top quark, it may not be so terrible. Another feature is the occurrence of colour sextet down-type quarks $S$. Provided the extra particles are heavy in some sense, the $X$ which can decay weakly into conventional down quarks, and $S$ which might provide a small admixture to nucleon states, might impinge on newly discovered narrow (multiquark?) resonances that fleetingly appear in some experiments. It is these differences from the standard picture that add spice to our scheme but also make it vulnerable.

The allocation of fermionic states to $\zeta$-polynomials through a superfield $\Psi$, a function of $x$ and $\zeta$, is studied in some detail in section 2, with emphasis on weak isospin assignments of the combinations and their count supplied in section 3. (However, gory 
details are relegated to an appendix.) Section 4 deals with the Higgs superfield $\Phi$ and its interaction with $\Psi$. Finally, the last section is an attempt to work out flavour matrix of the quarks and leptons engendered by eight real vacuum Higgs field components and a ninth complex component that is natural source of CP-violation. We then try to do some numerical work with those expectation values in order to obtain the $V_{U D}$ and $V_{L N}$ mixing matrices; because there are a lot of extra states we have to make some (frail) assumptions so as to achieve some simplification, and anyone with experience in mixing matrices will know that there is obviously quite a lot that could be improved here. But it is a start.

\section{Superfields}

Our scheme is founded on a set of five complex $\zeta$ for reasons discussed in an earlier paper. Four is too few and six is far too many, so our construction does accord with $\mathrm{SU}(5)$ and $\mathrm{SO}(10)$ grand unified models - and this is probably no accident. Recapitulating previous work, the assigments of these property coordinates for charge and fermion number are: $Q\left(\zeta^{0}, \zeta^{1}, \zeta^{2}, \zeta^{3}, \zeta^{4}\right)=(0,1 / 3,1 / 3,1 / 3,-1)$ and $F\left(\zeta^{0}, \zeta^{1}, \zeta^{2}, \zeta^{3}, \zeta^{4}\right)=$ $(1,-1 / 3,-1 / 3,-1 / 3,1)$. 'Neutrinicity' is tied to $\zeta^{0}$, 'chromicity' to $\zeta^{i}$ where $i=1,2,3$ are the three colours, and charged 'leptonicity' to $\zeta^{4}$ in building up properties/flavours. These superfields $\Psi$ and $\Phi$ (both overall Bose, note) are expanded in odd and even powers of $\zeta, \bar{\zeta}$ respectively. Thus the fermion field components sit in odd sectors and the bose field components lie in the even sectors of these expansions:

$$
\begin{aligned}
& \Phi(x, \zeta, \bar{\zeta})=\sum_{\text {even } r+\bar{r}}(\bar{\zeta})^{\bar{r}} \phi_{(\bar{r}),(r)}(x)(\zeta)^{r}, \\
& \Psi_{\alpha}(x, \zeta, \bar{\zeta})=\sum_{\text {odd } r+\bar{r}}(\bar{\zeta})^{\bar{r}} \psi_{\alpha(\bar{r}),(r)}(x)(\zeta)^{r} .
\end{aligned}
$$

Having said that, the behaviours under the Lorentz group of $\Phi$ and $\Psi_{\alpha}$ are very different, so these superfields are to be regarded as distinct and ought not to be combined in any way. A nice way of depicting the component fields is to draw up a six-by-six magic square and look at the entries therein.

There are so many fermionic $\psi$ entries (namely 512) that they invite pruning. We do so by tying reflection about the main diagonal to conjugation, a sensible choice since $\zeta \leftrightarrow \bar{\zeta}$, or

$$
\psi_{\alpha(\bar{r}),(r)}^{(c)}=\psi_{\alpha(r)(\bar{r})}, \quad \phi_{(\bar{r}),(r)}=\phi_{(r)(\bar{r})}^{\dagger},
$$

but also we apply another constraint that superfields are selfdual in some way, corresponding to reflection about the cross-diagonal. (The dual operation ${ }^{\times}$leaves quantum numbers intact.) One particular way is to require $\Psi$ to be anti-selfdual as this exorcises some embarrassing states hiding in the square such as $\bar{\zeta}_{0} \bar{\zeta}_{4} \zeta^{1} \zeta^{2} \zeta^{3}$ and $\bar{\zeta}_{4} \zeta^{0} \zeta^{1} \zeta^{2} \zeta^{3}$ which possess $F=-3$ and $Q=2$ respectively. This condition also disposes of a neutrino-like state in the upper right corner too, $\zeta^{0} \zeta^{1} \zeta^{2} \zeta^{3} \zeta^{4}$, which may be a mixed blessing. 
From hereon we focus on fermions that are colour singlets or triplets since we want to concenrate on quarks and leptons. Colour sextets do arise as well but we shall ignore them for now. Placing the up $U, D, L, N$ states in the magic square produces the following table $\ddagger$ :

\begin{tabular}{|l||c|c|c|c|c|c|}
\hline$r \backslash \bar{r}$ & 0 & 1 & 2 & 3 & 4 & 5 \\
\hline 0 & & $L_{1}, N_{1}, D_{5}^{c}$ & $L_{2,3}, N_{2,3}, D_{3,6,7}^{c}, U_{3}^{c}$ & $L_{5}^{c}, D_{1}, U_{1}$ & & $L_{6}^{c}, D_{2}, U_{2}$ \\
1 & $*$ & $*$ & $*$ & $L_{4}, N_{4}, D_{4,8}^{c}, U_{4}^{c}$ & & - \\
2 & & $*$ & $*$ & - & \\
3 & $*$ & $*$ & $*$ & $*$ & $*$ & - \\
4 & & $*$ & & & $*$ \\
5 & $*$ & & $*$ & & & \\
\hline
\end{tabular}

The full set of superfield components are normalized and listed in the appendix, from which one may verify the entries above (- means part of the dual, while ${ }^{*}$ means complex conjugate about the diagonal). Before turning to the Higgs superfield $\Phi$, we should mention that the adjoint of $\Psi$ has to be defined with appropriate sign changes. For example with only one $\zeta$, if one writes $\Psi_{\alpha}=\bar{\zeta} \psi_{\alpha}+\psi_{\alpha}^{c} \zeta$, then one should take $\bar{\Psi}^{\alpha}=-\bar{\psi}^{\alpha} \zeta+\bar{\zeta} \bar{\psi}^{c}$. This convention ensures that $\bar{\Psi} \Psi=\bar{\zeta} \zeta\left(\bar{\psi} \psi+\bar{\psi}^{c} \psi^{c}\right)=2 \bar{\zeta} \zeta \bar{\psi} \psi$, so that integration over $\zeta, \bar{\zeta}$ yields $2 \bar{\psi} \psi$. (Without that sign change there would have been a cancellation between the field and charge conjugate contributions.)

There are two more colour triplet quarks of interest; they are the companions of $U, D$ in the third and fourth generation, namely: $X_{3} \sim \bar{\zeta}_{0} \bar{\zeta}_{1} \zeta^{4}\left(1+\bar{\zeta}_{2} \zeta^{2} \bar{\zeta}_{3} \zeta^{3}\right) / 2$ and $X_{4} \sim \bar{\zeta}_{0} \bar{\zeta}_{1} \zeta^{4}\left(\bar{\zeta}_{2} \zeta^{2}+\bar{\zeta}_{3} \zeta^{3}\right) / 2$. More about them anon.

\section{Weak isopin assignments and new particle signatures}

Just from the way the anticommuting model is constructed, it will not have escaped the reader that leptonicity coordinates $\zeta^{0}, \zeta^{4}$ comprise a weak isodoublet, whereas the chromicity coordinates $\zeta^{i}$ are weak isosinglets. By the same reasoning, the conjugates $-\bar{\zeta}_{4}, \bar{\zeta}_{0}$ may be expected to form an isodoublet. Quantifying this algebraically, define the raising and lowering operators:

$$
T_{+}=\zeta^{0} \partial_{4}-\bar{\zeta}_{4} \bar{\partial}^{0}, \quad T_{-}=\zeta^{4} \partial_{0}-\bar{\zeta}_{0} \bar{\partial}^{4},
$$

where $\partial_{\mu} \equiv \partial / \partial \zeta^{\mu}$ and $\bar{\partial}^{\mu} \equiv \partial / \partial \bar{\zeta}_{\mu}$ are independent derivatives because $\zeta$ is complex. Hence the third component of weak isospin is given by

$$
2 T_{3}=\left[T_{+}, T_{-}\right]=\zeta^{0} \partial_{0}-\zeta^{4} \partial_{4}+\bar{\zeta}_{4} \bar{\partial}^{4}-\bar{\zeta}_{0} \bar{\partial}^{0} .
$$

It is thereby easy to establish that the polynomial $\left(\bar{\zeta}_{0} \zeta^{0}+\bar{\zeta}_{4} \zeta^{4}\right)$ and therefore its square $2 \bar{\zeta}_{0} \zeta^{0} \bar{\zeta}_{4} \zeta^{4}$ are weak isosinglets. Correspondingly, we have a weak isotriplet in the combinations $\left(-\bar{\zeta}_{4} \zeta^{0},\left(\bar{\zeta}_{0} \zeta^{0}-\bar{\zeta}_{4} \zeta^{4}\right) / \sqrt{2}, \bar{\zeta}_{0} \zeta^{4}\right)$.

$\ddagger$ we have relabelled components in an earlier article to conform with coming weak isospin assignments and have taken the opportunity to correct the $U$ quarks count from three to four. 
With regard to the heavy generations, $U, D$ together with $X$ make up a weak isotriplet - signifying a break from the standard model. Because $X$ carry charge $4 / 3$, they may decay weakly to the $D$ quarks. Colour singlet composites of $X_{3,4}$ with traditional quarks $U, D$ will produce new meson states $X \bar{U}$ and $X \bar{D}$ having charges -2 and -1 respectively; we can also envisage baryon states formed as colour singlet combinations of $X$ with two traditional quarks $U, D$ and one might look out for these too. Nor should it be forgotten that colour sextet quarks with $Q=-1 / 3$ and $F=1 / 3$ arise, being associated with the property combinations:

$$
S^{(k l)} \sim\left[\epsilon^{i j k} \zeta^{l}+\epsilon^{i j l} \zeta^{k}\right] \bar{\zeta}_{i} \bar{\zeta}_{j} / 4
$$

Thus a small part of proton could conceivably be the admixture $u_{k} u_{l} S^{(k l)}$, and so on. Perhaps combinations such as these may have a connection to ephemeral narrow width multiquark resonances found in some experiments.

Leaving such speculation aside, we are now in a position to summarise the weak isospin multiplets of quarks and leptons, based on the table and the explicit forms listed in the appendix. Firstly the leptons:

$$
\text { doublets : } \quad\left(\begin{array}{c}
N_{1,2,3,4} \\
L_{1,2,3,4}
\end{array}\right) ; \quad \text { singlets : } L_{5,6} \text {. }
$$

Secondly the colour triplet quarks:

$$
\text { doublets : }\left(\begin{array}{c}
U_{1,2} \\
D_{1,2}
\end{array}\right) ; \quad \text { triplets : }\left(\begin{array}{c}
U_{3,4} \\
D_{3,4} \\
X_{3,4}
\end{array}\right) ; \quad \text { singlets : } D_{5,6,7,8} \text {. }
$$

Thirdly, while we are at it, let us also list the vacuum expectation values of the antiselfdual Higgs superfield, again referring to the appendix:

singlets : $\mathcal{M}, \mathcal{A}+\mathcal{B}, \mathcal{C}, \mathcal{D}, \mathcal{E}+\mathcal{F}, \mathcal{G} ; \quad$ in doublet $: \mathcal{H}, \mathcal{H}^{*} ; \quad$ in triplet $: \mathcal{A}-\mathcal{B}, \mathcal{E}-\mathcal{F}$

\section{Strong interactions, gauge fields and couplings to matter}

Since the strong interactions are associated with colour the generators are readily expressed in terms of chromicity coordinates, namely we take the traceless part (to get $\mathrm{SU}(3))$ of

$$
T_{j}^{i}=\zeta^{i} \partial_{j}+\bar{\zeta}_{j} \bar{\partial}^{i}
$$

obeying the usual $\mathrm{U}(3)$ commutation relations,

$$
\left[T_{j}^{i}, T_{l}^{k}\right]=\delta_{j}^{k} T_{l}^{i}-\delta_{l}^{i} T_{j}^{k} .
$$

We thereby construct the matrix $\mathrm{A}=A_{i}^{j}\left(T_{j}^{i}-\delta_{j}^{i} T_{k}^{k} / 3\right)$ to contain the eight gluon fields $A$ in much the same way that $\mathrm{SU}(2)_{L}$ weak bosons are connected with the matrix

$$
\mathrm{W}=W^{3} T_{3}+\left(W^{+} T_{-}+W^{-} T_{+}\right) / \sqrt{2},
$$


where the weak isospin generators are given in eqns. (3) and (4).

It is quite straightforward to accommodate the standard model components in this scheme since (at least for the first two families) we are dealing with all the quarks and leptons one is accustomed to within the set $\left(U_{1,2}, D_{1,2}, N_{1,2}, L_{1,2}\right)$. The only care needed is to ensure that left and right fields are distinguished by their isospin and hypercharge assignments. There are two ways to do this: one can either assume that the zeta coordinates for matter are specifically tied to full Dirac fields $\Psi$ and introduce appropriate chirality projection operators $P_{ \pm}=\left(1 \pm i \gamma_{5}\right) / 2$, or one can presume that $\Psi$ is a left-handed to begin with $\S$ and associate generators involving $\zeta$ with left chirality but those involving $\bar{\zeta}$ with right chirality. In the first approach for gauging the standard $\mathrm{SU}(3) \times \mathrm{SU}(2)_{L} \times \mathrm{U}(1)$ we would construct the covariant derivative acting on Dirac $\Psi$ :

$$
\gamma \cdot D \equiv \gamma \cdot\left[\partial-i g \mathrm{~W} P_{-}-i g^{\prime} B\left(Y_{-} P_{-}+Y_{+} P_{+}\right)\right]
$$

where

$$
Y_{-}=-\left(\zeta^{0} \partial_{0}+\bar{\zeta}_{0} \bar{\partial}^{0}+\zeta^{4} \partial_{4}+\bar{\zeta}_{4} \bar{\partial}^{4}\right) / 2, \quad Y_{+}=-\left(\zeta^{4} \partial_{4}+\bar{\zeta}_{4} \bar{\partial}^{4}\right)
$$

In the second approach where $\psi$ is left-handed, we would take only one half of the isospin operators, namely $\mathrm{T}_{+}=\zeta^{0} \partial_{4}, \mathrm{~T}_{-}=\zeta^{4} \partial_{0}, \mathrm{~T}_{3}=\left(\zeta^{0} \partial_{0}-\zeta^{4} \partial_{4}\right) / 2$ and correspondingly $\mathrm{Y}=-\left(\zeta^{0} \partial_{0}+\zeta^{4} \partial_{4}\right) / 2-\bar{\zeta}_{4} \bar{\partial}^{4}$. (This guarantees that the right-handed particle fields are automatically weak isosinglets and is more elegant than the first approach.) Anyhow, with this halved set of generators of the second approach acting on $\Psi_{L}$ the covariant derivative reads

$$
\gamma \cdot D=\gamma \cdot\left[\partial-i g \mathrm{~W}-i g^{\prime} B \mathrm{Y}\right]
$$

These manoeuvres just beg the question as to why one cannot gauge a larger set of group generators. Of course one can, like any unified gauge model, but unfortunately nature has picked on the standard model subset, the vagary of picking on left-handed isospin being particularly quirky. Ultimately our anticommuting coordinate scheme invites us to gauge the full $\mathrm{Sp}(10)$ group for the 10 real anticommuting properties, but then we would be hard-pressed to explain where lie all the other gauge fields, not to mention the problem of handling their leptoquark components. Of course this difficulty is common to all unified schemes; the only virtue of ours is that the extra particles and states are severely circumscribed and from the point of view of 'economy' this is surely a distinct advantage; also we get a concrete realization of the group structure.

One last point concerns gauge anomalies. It is clear that for the lowest two families, which are common to our approach and the conventional one, there can be none, i.e. there is complete cancellation between leptons and quarks, per generation. Although we have not investigated what happens to the third generation which contains $U, D, X$ in a triplet and must be considered with some of the other states, it is fairly clear that the anomalies will disappear again for the standard model gauging because $\mathrm{SU}(3)$ is vectorial, $\mathrm{SU}(2)$ has no anomalies and the trace of the charge operator vanishes. As

$\S$ But remember that fields reflected about the diagonal contain the left-handed antiparticles so we are in effect including the right-handed particle states too. 
far as the unified gauge groups $\mathrm{SU}(5)$ or $\mathrm{Sp}(10)$ are concerned it would be surprising if the same thing did not happen again, as this scheme has a lot in common with $\mathrm{SO}(10)$ where we know there are no anomalies; for example the first row of the magic square is simply a 16-fold of $\mathrm{SO}(10)$; however we readily admit that the matter has not been defintively settled.

Finally, with regard to the Lagrangian for the gauge field, this can be obtained in the same way through the extended space-time-property metric, outlined in an earlier paper [1] for QED, but neglecting the gravitational part. It falls out of course as the non-abelian version of the Maxwell Lagrangian.

\section{Flavour mixing and mass matrices}

The coupling of the superfield $\Psi$ to the vacuum Hiigs superfield $\langle\Phi\rangle$ will induce flavour mixing. This arises via the interaction $4 \int d^{5} \zeta d^{5} \bar{\zeta} \bar{\Psi}\langle\Phi\rangle \Psi$, where we ignore an overall coupling constant for the purposes of this work (absorbing it in $\Phi$ ). Based on the assumption that the Higgs field is anti-selfdual like $\Psi$, only nine expectation values make an appearance, labelled $\mathcal{M}, \mathcal{A}, \ldots \mathcal{H}$ of which just the final one is complex. (Bear in mind that without antiduality there would be twice as many independent expectation values.) To see how to arrive at the results below we give an example, the flavour mixing of the first two charged leptons with one another, ignoring other components. First form the product $\bar{\Psi} \Psi$ and pick out the relevant parts, viz.

$$
\begin{aligned}
2 \bar{\Psi} \Psi \supset & \bar{L}_{1} L_{1} \bar{\zeta}_{4} \zeta^{4}\left(1-2 \bar{\zeta}_{0} \zeta^{0} \bar{\zeta}_{1} \zeta^{1} \bar{\zeta}_{2} \zeta^{2} \bar{\zeta}_{3} \zeta^{3}\right)+ \\
& \bar{L}_{2} L_{2} \bar{\zeta}_{4} \zeta^{4}\left(-2 \bar{\zeta}_{0} \zeta^{0} \bar{\zeta}_{1} \zeta^{1} \bar{\zeta}_{2} \zeta^{2} \bar{\zeta}_{3} \zeta^{3}\right)+ \\
& \left(\bar{L}_{1} L_{2}+\bar{L}_{2} L_{1}\right) \bar{\zeta}_{4} \zeta^{4}\left(\bar{\zeta}_{0} \zeta^{0}-\bar{\zeta}_{1} \zeta^{1} \bar{\zeta}_{2} \zeta^{2} \bar{\zeta}_{3} \zeta^{3}\right)+\cdots
\end{aligned}
$$

Then multiply by $\langle\Phi\rangle$ and pick out the zeta-product factor $\left(\bar{\zeta}_{\mu} \zeta^{\mu}\right)^{5} / 5$ !, needed for Berezin integration. One obtains the terms

$$
4 \int d^{5} \zeta d^{5} \bar{\zeta} \bar{\Psi}\langle\Phi\rangle \Psi \supset(2 \mathcal{M}+\mathcal{B}) \bar{L}_{1} L_{1}+2 \mathcal{M} \bar{L}_{2} L_{2}+(\mathcal{A}+\mathcal{D})\left(\bar{L}_{1} L_{2}+\bar{L}_{2} L_{1}\right)+\cdots
$$

and so on. Proceeding in this way, we can list the entire set of flavour mixing mass matrices $M_{i, j}$ that arise, where the range of indices, $i, j$ depends on the particle type. For charged leptons the hermitian matrix $M$ reads

$$
2 M(L) \rightarrow\left(\begin{array}{cccccc}
2 \mathcal{M}+\mathcal{B} & \mathcal{A}+\mathcal{D} & \mathcal{C}+\mathcal{F} & \mathcal{E}-\mathcal{G} & -\mathcal{H}^{*} & \mathcal{H}^{*} \\
\mathcal{A}+\mathcal{D} & 2 \mathcal{M} & -\mathcal{G} & \mathcal{C} & \mathcal{H}^{*} & 0 \\
\mathcal{C}+\mathcal{F} & -\mathcal{G} & 2(\mathcal{M}-\mathcal{E} / \sqrt{3}) & \mathcal{A}-2 \mathcal{C} / \sqrt{3} & 0 & 0 \\
\mathcal{E}-\mathcal{G} & \mathcal{C} & \mathcal{A}-2 \mathcal{C} / \sqrt{3} & 2 \mathcal{M} & 0 & 0 \\
-\mathcal{H} & \mathcal{H} & 0 & 0 & 2 \mathcal{M}+\mathcal{D} & \mathcal{A}+\mathcal{B} \\
\mathcal{H} & 0 & 0 & 0 & \mathcal{A}+\mathcal{B} & 2 \mathcal{M}
\end{array}\right)
$$


The neutrinos' matrix is smaller (just $4 \times 4$ ):

$$
2 M(N) \rightarrow\left(\begin{array}{cccc}
2 \mathcal{M}+\mathcal{A} & \mathcal{B}+\mathcal{D} & \mathcal{C}+\mathcal{E} & \mathcal{F}-\mathcal{G} \\
\mathcal{B}+\mathcal{D} & 2 \mathcal{M} & -\mathcal{G} & \mathcal{C} \\
\mathcal{C}+\mathcal{E} & -\mathcal{G} & 2(\mathcal{M}-\mathcal{F} / \sqrt{3}) & \mathcal{B}-2 \mathcal{C} / \sqrt{3} \\
\mathcal{F}-\mathcal{G} & \mathcal{C} & \mathcal{B}-2 \mathcal{C} / \sqrt{3} & 2 \mathcal{M}
\end{array}\right)
$$

as is that of the $U$-quarks:

$$
2 M(U) \rightarrow\left(\begin{array}{cccc}
2 \mathcal{M}+\mathcal{F} / \sqrt{3} & \mathcal{B}+\mathcal{C} / \sqrt{3} & -\mathcal{H}^{*} & 0 \\
\mathcal{B}+\mathcal{C} / \sqrt{3} & 2 \mathcal{M} & 0 & 0 \\
-\mathcal{H} & 0 & 2 \mathcal{M}+\mathcal{G} / \sqrt{3} & 2 \mathcal{C} / \sqrt{3} \\
0 & 0 & 2 \mathcal{C} / \sqrt{3} & 2 \mathcal{M}
\end{array}\right)
$$

but the $D$-quarks' $2 M(D)$ matrix is very complicated, being $8 \times 8$ :

$$
\left(\begin{array}{cccccccc}
2 \mathcal{M}+\frac{\mathcal{E}}{\sqrt{3}} & \mathcal{A}+\frac{\mathcal{C}}{\sqrt{3}} & -\mathcal{H}^{*} & 0 & \mathcal{H}^{*} & -\mathcal{H}^{*} & 0 & 0 \\
\mathcal{A}+\frac{\mathcal{C}}{\sqrt{3}} & 2 \mathcal{M} & 0 & 0 & \mathcal{H}^{*} & 0 & 0 & 0 \\
-\mathcal{H} & 0 & 2 \mathcal{M}+\frac{\mathcal{G}}{\sqrt{3}} & \frac{2 \mathcal{C}}{\sqrt{3}} & \frac{\mathcal{E}-\mathcal{F}}{\sqrt{6}}+\frac{\mathcal{A}-\mathcal{B}}{\sqrt{2}} & 0 & \frac{\mathcal{E}-\mathcal{F}}{\sqrt{3}} & \frac{\mathcal{B}-\mathcal{A}}{\sqrt{2}} \\
0 & 0 & \frac{2 \mathcal{C}}{\sqrt{3}} & 2 \mathcal{M} & \frac{2(\mathcal{F}-\mathcal{E})}{\sqrt{6}} & 0 & \mathcal{A}-\mathcal{B} & 0 \\
\mathcal{H} & \mathcal{H} & \frac{\mathcal{E}-\mathcal{F}}{\sqrt{6}}+\frac{\mathcal{A}-\mathcal{B}}{\sqrt{2}} & \frac{2(\mathcal{F}-\mathcal{E})}{\sqrt{6}} & 2 \mathcal{M}+\frac{\mathcal{C}}{\sqrt{3}} & \frac{\mathcal{E}+\mathcal{F}}{\sqrt{6}}+\frac{\mathcal{A}+\mathcal{B}}{\sqrt{2}} & \frac{2(\mathcal{G}+\mathcal{C})}{\sqrt{3}} & \mathcal{D}-\frac{\mathcal{G}}{\sqrt{3}} \\
-\mathcal{H} & 0 & 0 & 0 & \frac{\mathcal{E}+\mathcal{F}}{\sqrt{6}}+\frac{\mathcal{A}+\mathcal{B}}{\sqrt{2}} & 2 \mathcal{M} & -\frac{\mathcal{E}+\mathcal{F}}{\sqrt{3}} & \frac{\mathcal{A}+\mathcal{B}}{\sqrt{2}} \\
0 & 0 & \frac{\mathcal{E}-\mathcal{F}}{\sqrt{3}} & \mathcal{A}-\mathcal{B} & \frac{2(\mathcal{G}+\mathcal{C})}{\sqrt{3}} & -\frac{\mathcal{E}+\mathcal{F}}{\sqrt{3}} & 2 \mathcal{M}-\mathcal{D} & -\frac{2 \mathcal{C}}{\sqrt{6}} \\
0 & 0 & \frac{\mathcal{B}-\mathcal{A}}{\sqrt{2}} & 0 & \mathcal{D}-\frac{\mathcal{G}}{\sqrt{3}} & \frac{\mathcal{A}+\mathcal{B}}{\sqrt{2}} & -\frac{2 \mathcal{C}}{\sqrt{6}} & 2 \mathcal{M}
\end{array}\right) .
$$

These flavour matrices may be diagonalised by unitary tranformations $V$ in the usual way: $m(u)=V(U) M(U) V^{\dagger}(U)$, etc. And of course $V_{U D} \equiv V^{\dagger}(U) V(D)$ is also unitary in the standard picture because all quarks are weak isodoublets. But this is not what occurs in our scheme; to see what happens, focus on the charged weak interactions mediated by $W^{ \pm}$. Realizing that its coupling to the weak isotriplets differ by a factor of $\sqrt{2}$ from those of the isodoublets, we meet the terms

$$
W^{+}\left[\left(\bar{U}_{1} D_{1}+\bar{U}_{2} D_{2}\right)+\sqrt{2}\left(\bar{U}_{3} D_{3}+\bar{U}_{4} D_{4}+\bar{D}_{3} X_{3}+\bar{D}_{4} X_{4}\right)\right]+\text { h.c }
$$

which transcribe to the mass eigenvectors $v, \delta$ :

$$
\begin{aligned}
W^{+} \bar{v}_{j}\left[\left(V_{1 j}^{*}(U) V_{1 k}(D)\right.\right. & \left.\left.+V_{2 j}^{*}(U) V_{2 k}(D)\right)+\sqrt{2}\left(V_{3 j}^{*}(U) V_{4 k}(D)+V_{4 j}^{*}(U) V_{4 k}(D)\right)\right] \delta_{k} \\
& + \text { h.c. }+W D X \text { interaction - terms. }
\end{aligned}
$$

(Here the first three $v$ correspond to $c, u, t$ quarks and the first three $\delta$ to $s, d, b$ quarks.) No longer is the effective $V_{U D}$ matrix unitary. Depending on the amount of flavour mixing of weak isodoublets with isotriplets and isosinglets $D_{5, . .8}$, we anticipate that small departures from unitarity will arise; also weak interactions of $b$ and $t$ quarks will be somewhat larger than those of the first and second generations. The same remarks 
apply to the leptons in a modified manner: while the first four generations of $N, L$ are weak isodoublets, $L_{5,6}$ are isosinglets and it is their flavour mixing with the lighter $L \mathrm{~s}$ that may may induce a departure from unitarity of the $4 \mathrm{x} 4 V_{L N}$ matrix.

We are ready to make a stab at numerical values of masses and flavour mixings more in an attempt to understand the mechanics than in trying to accurately reproduce known mixings. It is futile to aim at exact figures for the known twelve lepton and quark masses plus all their CKM-type mixings with only nine parameters, $\mathcal{A}$ to $\mathcal{H}$, for we are looking at figures which range from about $10^{-2} \mathrm{eV}$ (for neutrinos) to $10^{11} \mathrm{eV}$ (for the top quark), in other words thirteen orders of magnitude, with many masses interspersed in that range. It is more likely that we can achieve a fit to data with a Higgs superfield that is not self-dual as that doubles the numbers of degrees of fitting freedom, but we leave that to future work. For the present we shall simply ignore the leptons and stick with the four $U$ quarks and eight $D$ quarks as getting even these to be roughly right represents a modicum of progress.

We have not searched through the whole of parameter space in presenting the following set of values; rather our aim has been to get acceptable values for $m_{u}, m_{d}, m_{c}, m_{s}, m_{t}, m_{b}$ and ensure that the other quark masses are heavy, in regions not yer fully explored. The hope is that this can yield flavour mixing matrices $\|$ that are not outright silly. Here is what we have adopted in units of $\mathrm{MeV}$ :

$$
\begin{aligned}
& \mathcal{M}=3, \mathcal{C}=-168000, \mathcal{B}=78+\mathcal{C} / \sqrt{3}, \mathcal{A}=5-\mathcal{C} / \sqrt{3} \\
& \mathcal{D}=7700000, \mathcal{E}=600, \mathcal{F}=2600, \mathcal{G}=70000, \mathcal{H}=100
\end{aligned}
$$

and thereby obtained

$$
m_{u}=2, m_{c}=1510, m_{t}=-175000, m_{d}=6, m_{s}=354, m_{b}=-4990 .
$$

[Negative mass eigenvalues are not particularly worrying since they can be reversed by a $\gamma_{5}$ transformation on the eigenfields or equivalently a relative change in phase of the left and right handed chiral components.] These numbers are satisfactory and so too are the masses of the other quarks which lie in the range above $175 \mathrm{GeV}$. (Actually there are strong indications from the analysis that another D-quark may exist almost degenerate with the top.)

Less successful is our determination of the mixing angles; the mass diagonalised states of $U$-quarks are not too bad, being given by the $\mathrm{V}$-matrix:

$$
\left(\begin{array}{c}
c \\
u \\
t \\
v_{4} \\
\cdot
\end{array}\right)=\left(\begin{array}{ccccc}
0.9987 & 0.052 & 4 \times 10^{-6} & 5 \times 10^{-4} & \cdot \\
-0.052 & 0.9987 & 6 \times 10^{-10} & 3 \times 10^{-4} & \cdot \\
3.8 \times 10^{-4} & -1.7 \times 10^{-7} & 0.67 & 0.74 & \cdot \\
-3.4 \times 10^{-4} & -10^{-7} & 0.74 & -0.67 & \cdot \\
. & . & . & . & \cdot
\end{array}\right)\left(\begin{array}{c}
U_{1} \\
U_{2} \\
U_{3} \\
U_{4} \\
\cdot
\end{array}\right),
$$

$\|$ We have not bothered to include a phase in the complex field $\mathcal{H}$ which is the source of CP-violation as it introduces another layer of complexity. 
but the $D$-quarks' V-matrix is more problematic:

$$
\left(\begin{array}{c}
s \\
d \\
b \\
\delta_{4} \\
\cdot
\end{array}\right)=\left(\begin{array}{ccccc}
0.9997 & 0.014 & 4 \times 10^{-6} & -5 \times 10^{-4} & . \\
-0.014 & 0.9999 & 6 \times 10^{-8} & 7 \times 10^{-6} & \cdot \\
0.02 & 0.00034 & -1.5 \times 10^{-4} & 3.8 \times 10^{-5} & . \\
-3.8 \times 10^{-4} & -10^{-7} & -0.67 & -0.74 & \cdot \\
. & . & . & . & .
\end{array}\right)\left(\begin{array}{c}
D_{1} \\
D_{2} \\
D_{3} \\
D_{4} \\
\cdot
\end{array}\right)
$$

because it means that the $b$ quark is largely $D_{6}$ (an electroweak singlet!) which is very far from conventional wisdom. This is a definite failure of our parameter choice. Given that we have abandoned any attempt at fitting the (light) lepton masses, as is clear from the scales above, we will not apologize any more. If one were to double the Higgs field expectation values by discarding selfduality we would be in better shape all round, but that would require a search through an 18-parameter space which is non-trivial!

In summary we reiterate the places where we differ from the standard model.

- We have at least four generations of quarks and leptons. Of the six charged leptons two are weak isosinglets, and of the eight $D$-quarks four are weak isosinglets.

- The top and bottom quarks belong to a weak isotriplet, together with another quark which we have designated $X$.

- The flavour mixing induced by the super-Higgs components can result in a $V_{U D}$ which is not quite unitary because the third generation is an isotriplet; this would be a good experimental test of our scheme.

- Colour sextet quarks with $F=1 / 3, Q=-1 / 3$ arise in property combinations $(\bar{\zeta})^{2} \zeta$ where the zeta only carry chromicity. If baryons carry small admixtures of these sextets in conjunction with ordinary $u, d$ quarks this might help explain features of newly discovered narrow width resonances that are interpreted as multiquarks.

\section{Appendix - Normalized superfields}

Here we list the anti-selfdual components of the superfields $\Psi$ and $\Phi$, arranged so that after Berezin integration they are properly normalized; our convention is that $\int d^{5} \zeta d^{5} \bar{\zeta}\left(\bar{\zeta}_{\mu} \zeta^{\mu}\right) \equiv-5$ ! We only list relevant parts that contain leptons and colour triplet quarks, these being fields of primary interest, and start with red $U$-quarks; the latter are associated with (red) label 1 in the $\zeta$ expansion - not to be confused with the flavour counting subscripts carried by $U$ :

$$
\begin{aligned}
2 \Psi_{U} \supset 2 \Psi_{U \text { red }}= & {\left[\bar{\zeta}_{3} \bar{\zeta}_{2} \bar{\zeta}_{0} U_{1}+U_{1}^{c} \zeta^{0} \zeta^{2} \zeta^{3}\right]\left(1+\bar{\zeta}_{4} \zeta^{4} \bar{\zeta}_{1} \zeta^{1}\right)+} \\
& {\left[\bar{\zeta}_{3} \bar{\zeta}_{2} \bar{\zeta}_{0} U_{2}+U_{2}^{c} \zeta^{0} \zeta^{2} \zeta^{3}\right]\left(\bar{\zeta}_{1} \zeta^{1}+\bar{\zeta}_{4} \zeta^{4}\right)+} \\
& {\left[\bar{\zeta}_{0} \zeta^{1} \zeta^{4} U_{3}+U_{3}^{c} \bar{\zeta}_{4} \bar{\zeta}_{1} \zeta^{0}\right]\left(1+\bar{\zeta}_{2} \zeta^{2} \bar{\zeta}_{3} \zeta^{3}\right)+} \\
& {\left[\bar{\zeta}_{0} \zeta^{1} \zeta^{4} U_{4}+U_{4}^{c} \bar{\zeta}_{4} \bar{\zeta}_{1} \zeta^{0}\right]\left(\bar{\zeta}_{2} \zeta^{2}+\bar{\zeta}_{3} \zeta^{3}\right) . }
\end{aligned}
$$

Because this is the first time we encounter it, let us also spell out the adjoint components, taking due care with sign changes:

$$
2 \bar{\Psi}_{U} \supset 2 \bar{\Psi}_{U \text { red }}=\left[\bar{U}_{1} \zeta^{0} \zeta^{2} \zeta^{3}-\bar{\zeta}_{3} \bar{\zeta}_{2} \bar{\zeta}_{0} \bar{U}_{1}^{c}\right]\left(1+\bar{\zeta}_{4} \zeta^{4} \bar{\zeta}_{1} \zeta^{1}\right)+
$$


Mass Matrix

$$
\begin{aligned}
& {\left[\bar{U}_{2} \zeta^{0} \zeta^{2} \zeta^{3}-\bar{\zeta}_{3} \bar{\zeta}_{2} \bar{\zeta}_{0} \bar{U}_{2}^{c}\right]\left(\bar{\zeta}_{1} \zeta^{1}+\bar{\zeta}_{4} \zeta^{4}\right)+} \\
& {\left[\bar{U}_{3} \bar{\zeta}_{4} \bar{\zeta}_{1} \zeta^{0}-\bar{\zeta}_{0} \zeta^{1} \zeta^{4} \bar{U}_{3}^{c}\right]\left(1+\bar{\zeta}_{2} \zeta^{2} \bar{\zeta}_{3} \zeta^{3}\right)+} \\
& {\left[\bar{U}_{4} \bar{\zeta}_{4} \bar{\zeta}_{1} \zeta^{0}-\bar{\zeta}_{0} \zeta^{1} \zeta^{4} \bar{U}_{4}^{c}\right]\left(\bar{\zeta}_{2} \zeta^{2}+\bar{\zeta}_{3} \zeta^{3}\right) .}
\end{aligned}
$$

One may readily check that $\int d^{5} \zeta d^{5} \bar{\zeta}\left(\bar{\Psi}_{U \text { red }} \Psi_{U \text { red }}\right)=\left(\bar{U}_{1} U_{1}+\bar{U}_{2} U_{2}+\bar{U}_{3} U_{3}+\bar{U}_{4} U_{4}\right)$.

Next we list the red down-type quark field components, again designated by (red) label 1 so far as the $\zeta$ coordinates are concerned. It contains twice as many pieces as the up-type quarks:

$$
\begin{aligned}
2 \bar{\Psi}_{D} \supset 2 \bar{\Psi}_{D \text { red }}= & {\left[\bar{\zeta}_{3} \bar{\zeta}_{2} \bar{\zeta}_{4} D_{1}+D_{1}^{c} \zeta^{4} \zeta^{2} \zeta^{3}\right]\left(1+\bar{\zeta}_{0} \zeta^{0} \bar{\zeta}_{1} \zeta^{1}\right)+} \\
& {\left[\bar{\zeta}_{3} \bar{\zeta}_{2} \bar{\zeta}_{4} D_{2}+D_{2}^{c} \zeta^{4} \zeta^{2} \zeta^{3}\right]\left(\bar{\zeta}_{1} \zeta^{1}+\bar{\zeta}_{0} \zeta^{0}\right)+} \\
& {\left[\bar{\zeta}_{1} D_{3}^{c}+D_{3} \zeta^{1}\right]\left(\bar{\zeta}_{0} \zeta^{0}-\bar{\zeta}_{4} \zeta^{4}\right)\left(1+\bar{\zeta}_{2} \zeta^{2} \bar{\zeta}_{3} \zeta^{3}\right) / \sqrt{2}+} \\
& {\left[\bar{\zeta}_{1} D_{4}^{c}+D_{4} \zeta^{1}\right]\left(\bar{\zeta}_{2} \zeta^{2}+\bar{\zeta}_{3} \zeta^{3}\right)\left(\bar{\zeta}_{0} \zeta^{0}-\bar{\zeta}_{4} \zeta^{4}\right) / \sqrt{2}+} \\
& {\left[\bar{\zeta}_{1} D_{5}^{c}+D_{5} \zeta^{1}\right]\left(1-\bar{\zeta}_{2} \zeta^{2} \bar{\zeta}_{3} \zeta^{3} \bar{\zeta}_{0} \zeta^{0} \bar{\zeta}_{4} \zeta^{4}\right)+} \\
& {\left[\bar{\zeta}_{1} D_{6}^{c}+D_{6} \zeta^{1}\right]\left(\bar{\zeta}_{0} \zeta^{0}+\bar{\zeta}_{4} \zeta^{4}\right)\left(1-\bar{\zeta}_{2} \zeta^{2} \bar{\zeta}_{3} \zeta^{3}\right) / \sqrt{2}+} \\
& {\left[\bar{\zeta}_{1} D_{7}^{c}+D_{7} \zeta^{1}\right]\left(\bar{\zeta}_{2} \zeta^{2}+\bar{\zeta}_{3} \zeta^{3}\right)\left(1-\bar{\zeta}_{0} \zeta^{0} \bar{\zeta}_{4} \zeta^{4}\right) / \sqrt{2}+} \\
& {\left[\bar{\zeta}_{1} D_{8}^{c}+D_{8} \zeta^{1}\right]\left(\bar{\zeta}_{0} \zeta^{0} \bar{\zeta}_{4} \zeta^{4}-\bar{\zeta}_{2} \zeta^{2} \bar{\zeta}_{3} \zeta^{3}\right) . }
\end{aligned}
$$

Note that the last four components are weak isosinglets. Here again, $\int d^{5} \zeta d^{5} \bar{\zeta}\left(\bar{\Psi}_{D \text { red }} \Psi_{D \text { red }}\right)=\left(\bar{D}_{1} D_{1}+\bar{D}_{2} D_{2}+\bar{D}_{3} D_{3}+\bar{D}_{4} D_{4}+\bar{D}_{5} D_{5}+\bar{D}_{6} D_{6}+\bar{D}_{7} D_{7}+\bar{D}_{8} D_{8}\right)$.

Turning to leptons, begin with the four neutrinos contained in

$$
\begin{aligned}
2 \Psi_{N}= & {\left[\bar{\zeta}_{0} N_{1}+N_{1}^{c} \zeta^{0}\right]\left(1-\bar{\zeta}_{4} \zeta^{4} \bar{\zeta}_{1} \zeta^{1} \bar{\zeta}_{2} \zeta^{2} \bar{\zeta}_{3} \zeta^{3}\right)+} \\
& {\left[\bar{\zeta}_{0} N_{2}+N_{2}^{c} \zeta^{0}\right]\left(\bar{\zeta}_{4} \zeta^{4}-\bar{\zeta}_{1} \zeta^{1} \bar{\zeta}_{2} \zeta^{2} \bar{\zeta}_{3} \zeta^{3}\right)+} \\
& {\left[\bar{\zeta}_{0} N_{3}+N_{3}^{c} \zeta^{0}\right]\left(\bar{\zeta}_{i} \zeta^{i}-\bar{\zeta}_{4} \zeta^{4} \bar{\zeta}_{j} \zeta^{j} \bar{\zeta}_{k} \zeta^{k} / 2\right) / \sqrt{3}+} \\
& {\left[\bar{\zeta}_{0} N_{4}+N_{4}^{c} \zeta^{0}\right]\left(\bar{\zeta}_{i} \zeta^{i} \bar{\zeta}_{4} \zeta^{4}-\bar{\zeta}_{j} \zeta^{j} \bar{\zeta}_{k} \zeta^{k} / 2\right) / \sqrt{3} }
\end{aligned}
$$

and then write out the charged leptons (very similar except for an extra two entries that are weak isospin singlets):

$$
\begin{aligned}
2 \Psi_{L}= & {\left[\bar{\zeta}_{4} L_{1}+L_{1}^{c} \zeta^{4}\right]\left(1-\bar{\zeta}_{0} \zeta^{0} \bar{\zeta}_{1} \zeta^{1} \bar{\zeta}_{2} \zeta^{2} \bar{\zeta}_{3} \zeta^{3}\right)+} \\
& {\left[\bar{\zeta}_{4} L_{2}+L_{2}^{c} \zeta^{4}\right]\left(\bar{\zeta}_{0} \zeta^{0}-\bar{\zeta}_{1} \zeta^{1} \bar{\zeta}_{2} \zeta^{2} \bar{\zeta}_{3} \zeta^{3}\right)+} \\
& {\left[\bar{\zeta}_{0} L_{3}+L_{3}^{c} \zeta^{0}\right]\left(\bar{\zeta}_{i} \zeta^{i}-\bar{\zeta}_{0} \zeta^{0} \bar{\zeta}_{j} \zeta^{j} \bar{\zeta}_{k} \zeta^{k} / 2\right) / \sqrt{3}+} \\
& {\left[\bar{\zeta}_{4} L_{4}+L_{4}^{c} \zeta^{4}\right]\left(\bar{\zeta}_{i} \zeta^{i} \bar{\zeta}_{0} \zeta^{0}-\bar{\zeta}_{j} \zeta^{j} \bar{\zeta}_{k} \zeta^{k} / 2\right) / \sqrt{3}+} \\
& {\left[\bar{\zeta}_{3} \bar{\zeta}_{2} \bar{\zeta}_{1} L_{5}^{c}+L_{5} \zeta^{1} \zeta^{2} \zeta^{3}\right]\left(1+\bar{\zeta}_{0} \zeta^{0} \bar{\zeta}_{4} \zeta^{4}\right)+} \\
& {\left[\bar{\zeta}_{3} \bar{\zeta}_{2} \bar{\zeta}_{1} L_{6}^{c}+L_{6} \zeta^{1} \zeta^{2} \zeta^{3}\right]\left(\bar{\zeta}_{0} \zeta^{0}+\bar{\zeta}_{4} \zeta^{4}\right) . }
\end{aligned}
$$

Altogether one may verify that $\int d^{5} \zeta d^{5} \bar{\zeta}\left(\bar{\Psi}_{N} \Psi_{N}+\bar{\Psi}_{L} \Psi_{L}\right)=\left(\bar{N}_{1} N_{1}+\bar{N}_{2} N_{2}+\bar{N}_{3} N_{3}+\right.$ $\left.\bar{N}_{4} N_{4}+\bar{L}_{1} L_{1}+\bar{L}_{2} L_{2}+\bar{L}_{3} L_{3}+\bar{L}_{4} L_{4}+\bar{L}_{5} L_{5}+\bar{L}_{6} L_{6}\right)$, which is perfectly satisfactory.

Finally we spell out the parts of the Higgs superfield that have $Q=F=0$. Having assumed anti-selfduality these components read:

$$
\begin{aligned}
2\langle\Phi\rangle= & \mathcal{M}\left(1-\bar{\zeta}_{0} \zeta^{0} \bar{\zeta}_{1} \zeta^{1} \bar{\zeta}_{2} \zeta^{2} \bar{\zeta}_{3} \zeta^{3} \bar{\zeta}_{4} \zeta^{4}\right)+\mathcal{A}\left(\bar{\zeta}_{0} \zeta^{0}-\bar{\zeta}_{1} \zeta^{1} \bar{\zeta}_{2} \zeta^{2} \bar{\zeta}_{3} \zeta^{3} \bar{\zeta}_{4} \zeta^{4}\right)+ \\
& \mathcal{B}\left(\bar{\zeta}_{4} \zeta^{4}-\bar{\zeta}_{1} \zeta^{1} \bar{\zeta}_{2} \zeta^{2} \bar{\zeta}_{3} \zeta^{3} \bar{\zeta}_{0} \zeta^{0}\right)+\mathcal{C}\left(\bar{\zeta}_{i} \zeta^{i}-\bar{\zeta}_{0} \zeta^{0} \bar{\zeta}_{4} \zeta^{4} \bar{\zeta}_{j} \zeta^{j} \bar{\zeta}_{k} \zeta^{k} / 2\right) / \sqrt{3}+
\end{aligned}
$$




$$
\begin{aligned}
& \mathcal{D}\left(\bar{\zeta}_{0} \zeta^{0} \bar{\zeta}_{4} \zeta^{4}-\bar{\zeta}_{1} \zeta^{1} \bar{\zeta}_{2} \zeta^{2} \bar{\zeta}_{3} \zeta^{3}\right)+\mathcal{E}\left(\bar{\zeta}_{i} \zeta^{i} \bar{\zeta}_{0} \zeta^{0}-\bar{\zeta}_{4} \zeta^{4} \bar{\zeta}_{j} \zeta^{j} \bar{\zeta}_{k} \zeta^{k} / 2\right) / \sqrt{3}+ \\
& \mathcal{F}\left(\bar{\zeta}_{i} \zeta^{i} \bar{\zeta}_{4} \zeta^{4}-\bar{\zeta}_{0} \zeta^{0} \bar{\zeta}_{j} \zeta^{j} \bar{\zeta}_{k} \zeta^{k} / 2\right) / \sqrt{3}+ \\
& \mathcal{G}\left(\bar{\zeta}_{i} \zeta^{i} \bar{\zeta}_{j} \zeta^{j} / 2-\bar{\zeta}_{4} \zeta^{4} \bar{\zeta}_{0} \zeta^{0} \bar{\zeta}_{k} \zeta^{k}\right) / \sqrt{3}+ \\
& {\left[\mathcal{H} \zeta^{1} \zeta^{2} \zeta^{3} \zeta^{4}+\mathcal{H}^{*} \bar{\zeta}_{4} \bar{\zeta}_{3} \bar{\zeta}_{2} \bar{\zeta}_{1}\right]\left(1-\bar{\zeta}_{0} \zeta^{0}\right)}
\end{aligned}
$$

Except for $\mathcal{H}$ all other expectation values are real. In fact it is the $\mathcal{H}$ components that are responsible for CP-violation in this scheme. Checking it all out, $\int d^{5} \zeta d^{5} \bar{\zeta} 2\langle\Phi\rangle^{2}=$ $\mathcal{M}^{2}+\mathcal{A}^{2}+\mathcal{B}^{2}+\mathcal{C}^{2}+\mathcal{D}^{2}+\mathcal{E}^{2}+\mathcal{F}^{2}+\mathcal{G}^{2}+2 \mathcal{H}^{*} \mathcal{H}$. We stress that relaxing selfduality for $\Phi$ will double the number of independent expectation values and provide more freedom in the mass matrices.

\section{References}

[1] Delbourgo R 2006 J. Phys. A39 5175

[2] Delbourgo R, Jarvis P D, Warner R C 1991 Aust. J. Phys. 44135

Delbourgo R, Twisk S, Zhang R 1988 Mod. Phys. Lett. A3 1073

Delbourgo R and White M 1990 Mod Phys. Lett. A5 355

[3] Jarvis P D and Dondi P H 1980 Z. Phys. C4 201

Jarvis P D and White M 1991 Phys. Rev. D43 4121 